\title{
In vitro characterization of neurite extension using induced pluripotent stem cells derived from lissencephaly patients with TUBA1A missense mutations
}

Yohei Bamba ${ }^{1,2}$, Tomoko Shofuda ${ }^{3}$, Mitsuhiro Kato ${ }^{4}$, Ritsuko K. Pooh ${ }^{5}$, Yoko Tateishi ${ }^{6}$, Jun-ichi Takanashi ${ }^{7}$ Hidetsuna Utsunomiya ${ }^{8}$, Miho Sumida ${ }^{2}$, Daisuke Kanematsu², Hiroshi Suemizu' ${ }^{9}$ Yuichiro Higuchi ${ }^{9}$, Wado Akamatsu', Denis Gallagher ${ }^{10,11,12}$, Freda D. Miller ${ }^{10,12,13}$, Mami Yamasaki ${ }^{14,15}$, Yonehiro Kanemura ${ }^{2,16}$ and Hideyuki Okano ${ }^{1 *}$

\begin{abstract}
Background: Lissencephaly, or smooth brain, is a severe congenital brain malformation that is thought to be associated with impaired neuronal migration during corticogenesis. However, the exact etiology of lissencephaly in humans remains unknown. Research on congenital diseases is limited by the shortage of clinically derived resources, especially for rare pediatric diseases. The research on lissencephaly is further limited because gyration in humans is more evolved than that in model animals such as mice. To overcome these limitations, we generated induced pluripotent stem cells (iPSCs) from the umbilical cord and peripheral blood of two lissencephaly patients with different clinical severities carrying alpha tubulin (TUBATA) missense mutations (Patient A, p.N329S; Patient B, p.R264C).

Results: Neural progenitor cells were generated from these iPSCS (iPSC-NPCs) using SMAD signaling inhibitors. These iPSC-NPCs expressed TUBATA at much higher levels than undifferentiated iPSCs and, like fetal NPCs, readily differentiated into neurons. Using these lissencephaly iPSC-NPCs, we showed that the neurons derived from the iPSCs obtained from Patient A but not those obtained from Patient B showed abnormal neurite extension, which correlated with the pathological severity in the brains of the patients.

Conclusion: We established iPSCs derived from lissencephaly patients and successfully modeled one aspect of the pathogenesis of lissencephaly in vitro using IPSC-NPCs and iPSC-derived neurons. The iPSCs from patients with brain malformation diseases helped us understand the mechanism underlying rare diseases and human corticogenesis without the use of postmortem brains.
\end{abstract}

Keywords: Lissencephaly, Induced pluripotent stem cells, TUBA1A, Neural progenitor cells

\footnotetext{
* Correspondence: hidokano@a2.keio.jp

'Department of Physiology, Keio University School of Medicine, 35

Shinanomachi, Shinjuku-ku, Tokyo 160-8582, Japan

Full list of author information is available at the end of the article
} 


\section{Background}

Dysfunction in the complicated cellular dynamics involved in the development of the cerebral cortex causes various congenital brain malformations during different stages of the development of the central nervous system (CNS) [1]. The two major modes of development in the human cerebral cortex are tangential and radial expansion. Tangential expansion evolves as the number of progenitor cells increases, as observed recently in the outer subventricular zone radial glia of gyrencephalic species including humans [2,3]. A decreased number or abnormal division of progenitor cells leads to microcephaly, or small brain. Radial expansion depends on neuronal migration in a basal to apical direction along the processes of the radial glia, and dysfunction in this process impairs corticogenesis in lissencephaly.

Lissencephaly, with disturbed cerebral cortical lamination, is one of the most severe brain malformations. In lissencephaly, the surface of the brain appears smooth owing to insufficient gyration, which is why this condition is called smooth brain. Lissencephaly is mainly caused by mutations in genes, many of which are involved in microtubule function. In addition to the genes encoding microtubule-associated proteins, alpha tubulin (TUBA1A) mutations have recently been shown to cause abnormal neuronal migration [4]. In humans, TUBA1A mutations have been identified in lissencephaly patients whose brains showed a smooth surface owing to severely impaired lamination of the cerebral cortex [4-6]. Lissencephaly in humans is an extremely rare disease, and lissencephaly patients often die within a few years, thus making it difficult to obtain viable patient-derived cells including neurons. This limitation has greatly restricted the complete elucidation of the etiology of lissencephaly in humans.

Therefore, to investigate the pathogenic mechanisms underlying lissencephaly in humans, we established induced pluripotent stem cells (iPSCs) from lissencephaly patients. Using neural progenitor cells and neurons generated from patient-derived iPSCs, we aimed to elucidate the disease pathology and to develop novel therapies.

\section{Methods}

\section{Generation of iPSCs}

Umbilical cords collected from Patient A with the p.N329S TUBA1A mutation (Fig. 1) and from vaginally delivered full-term fetal adnexa of healthy volunteers (male) were digested with collagenase I (Life Technologies, Carlsbad, CA, USA) and dispase (Life Technologies) for $30 \mathrm{~min}$ at $37^{\circ} \mathrm{C}$. The cells liberated from the tissue were then collected by centrifugation and seeded in T75 flasks in Dulbecco's modified Eagle's medium/nutrient mixture F-12 (DMEM/F12) (Sigma-Aldrich, St. Louis, MO, USA) containing $10 \%$ fetal bovine serum (FBS), 15 mM HEPES, and antibiotic-antimycotic solution (Life Technologies) [7]. Umbilical cord-derived stromal cells (UCCs) were passaged after 1 week and used for iPSC generation after 3-5 passages.
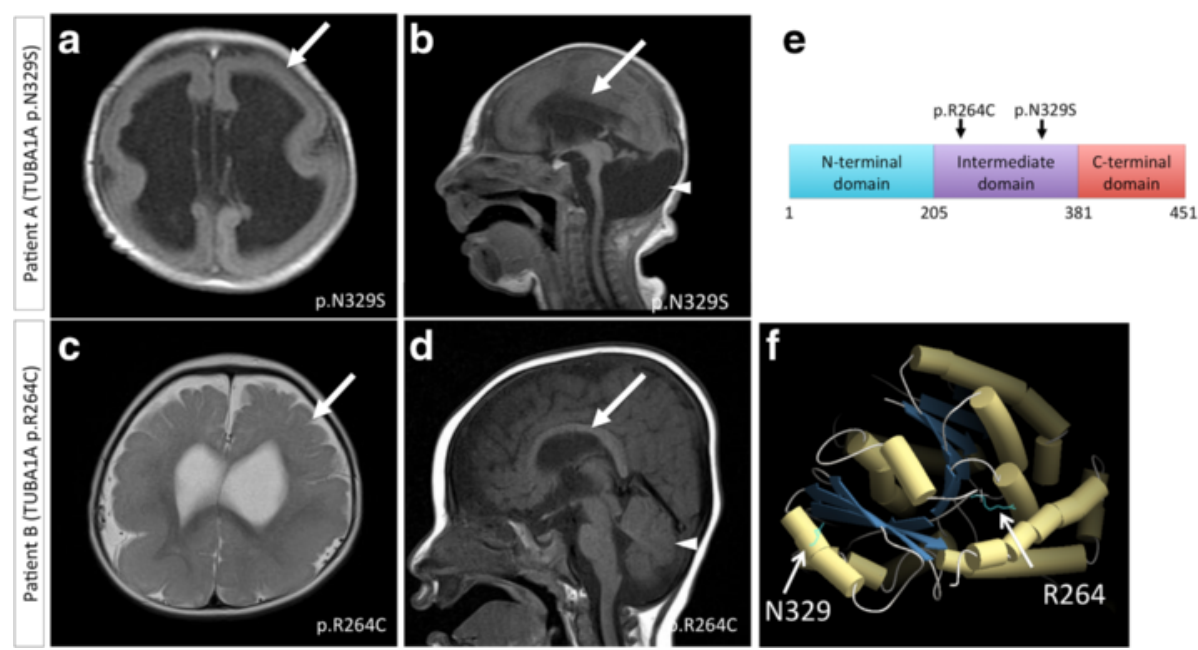

Fig. 1 Magnetic resonance imaging (MRI) of two patients. a, b Patient A (p.N329S TUBA1A mutation) shows lissencephaly with cerebellar hypoplasia. Thin cerebral mantle and agyric cerebral cortices (arrow in figure $\mathbf{a}$ ) are observed without an anterior-posterior gradient. The corpus callosum is not present (arrow in figure b). c, d Patient B (p.R264C TUBA1A mutation) shows pachygyria with a posterior-anterior gradient (arrow in figure c). Cerebellar and brain stem hypoplasia are not as clear as in Patient A (arrowhead in figure $\mathbf{d}$ ). The corpus callous is present (arrow in figure d). e Schematic structure of TUBA1A is represented based on a previous report [34]. Both missense mutations in TUBA1A were located in the intermediate domain of TUBA1A. $\mathbf{f}$ Three-dimensional structure of the TUBA1A protein. Helices are presented as cylinders. The arrows show the residue of each mutation. The p.N329S mutation was located on alpha-helix H10, which formed the interface with beta-tubulin. The p.R264C mutation was located between alpha-helix $\mathrm{H} 8$ and the beta sheet, which could be responsible for providing stability to the tertiary structure 
Peripheral blood mononuclear cells (PBMCs) from Patient B with the p.R264C TUBA1A mutation (Fig. 1) and from a healthy adult volunteer were isolated using Ficoll-Paque (GE Healthcare, Buckinghamshire, UK) according to the manufacturer's instructions. The isolated PBMCs were activated with immobilized anti-CD3 monoclonal antibodies (Orthoclone OKTR3 Injection, Janssen-Kyowa, Tokyo, Japan) and expanded in soluble interleukin (IL)-2-containing ALyS203 medium (NIPRO, Japan) with $10 \%$ FBS as previously described, with minor modifications [8]. In this study, we used the human iPSC line (201B7) [9] derived from human dermal fibroblasts from the facial dermis of a 36-year-old Caucasian female as the control (obtained from the RIKEN cell bank (Tsukuba, Japan)).

For iPSC generation, reprogramming episomal vectors (see below) were nucleofected using Amaxa Nucleofector I (a Neonatal Fibroblast Nucleofector Kit with the U-020 program for UCCs and a Human T cell Nucleofector Kit with the T-023 program for PBMCs; Lonza, Basel, Switzerland). Plasmids were used with combinations of pCXLE-hOct3/4-shp53, pCXLE-hUL, and pCXLE-hSK [10] for UCCs and PBMCs [11] or pCEB-hSKO and pCEB-hULG for UCCs. All the plasmids were graciously provided by Dr. Keisuke Okita and Prof. Shinya Yamanaka (Kyoto University, Kyoto, Japan). The subsequent procedures, including colony isolation and propagation, were performed as previously described [12]. The generated iPSCs were characterized using immunocytochemistry for the pluripotency-associated transcription factors OCT3/4 and NANOG; quantitative RT-PCR for the marker genes OCT3/4, SOX2, NANOG, ZFP42, and DNMT3B; and PCR according to protocols provided by the Center for Developmental Biology (RIKEN, http://www.cdb.riken.jp) and the Center for iPS Cell Research and Application (Kyoto University, https://www.cira.kyoto-u.ac.jp) to confirm that the episomal vectors did not persist.

The teratoma formation assay for the iPSCs was performed with subcutaneous transplantations into NOD/ Shi-SCID IL2R $\gamma$ null (NOG) mice $[12,13]$ in the Animal Facility of the CIEA (Central Institute for Experimental Animals, Kawasaki, Japan), in accordance with the guidelines of the Animal Care Committee at CIEA, and was approved by the Animal Care Committee at CIEA (No.11029A). The genomic sequences of TUBA1A were confirmed using previously reported methods [6].

The human embryonic stem cell line KhES1 [14] was propagated at Keio University in accordance with Japanese guidelines for the utilization of human embryonic stem cells with the approval of the Ministry of Education, Culture, Sports, Science and Technology of Japan and the ethical committee of Keio University. RNA samples from the KhES1 cells were isolated using an RNeasy minikit (QIAGEN, Valencia, CA, USA) and were used as a standard for quantitative RT-PCR. Summaries of the donor information and radiological images are shown in Table 1 and Fig. 1a-d, respectively. Both the TUBA1A mutations in these patients were located in the intermediate domain of TUBA1A, as shown in the schematic illustration in Fig. 1e. A karyotyping analysis was performed using conventional Giemsa staining and G-banding in all the generated iPSCs as described previously [12].

\section{Gene and protein analyses}

The sequences of the TUBA1A gene in this report are based on the sequences obtained from GenBank (Accession NM_006009). The protein structure of TUBA1A [15] was obtained from Protein Data Bank Japan (PDBj: 1JFF), and the illustration was generated using CueMol2 (http:/http://www.cuemol.org/en/).

\section{Neural induction by dual SMAD inhibition and neurosphere propagation}

The highly efficient dual inhibition of SMAD signaling was used for neural induction of the iPSCs [16]. Briefly, the iPSCs cultured in growth-factor-reduced (GFR) Matrigel (BD Biosciences, San Diego, CA, USA)-coated dishes were collected and suspended in low-attachment dishes (Primesurface, Sumitomo Bakelite, Tokyo, Japan) to form embryoid bodies (EBs). For neural induction, the EBs were subsequently cultured in DMEM/F12 containing $5 \%$ B27 supplement (Life Technologies), $5 \%$ N2 supplement (Life Technologies), $20 \mathrm{ng} / \mathrm{ml}$ recombinant human (rh) basic fibroblast growth factor (bFGF) (Wako, Osaka, Japan), $10 \mu \mathrm{M}$ SB431542 (SB; SigmaAldrich), and $1 \mu \mathrm{M}$ dorsomorphin (DSM; Wako) for 2 weeks under $5 \% \mathrm{O}_{2}$. To obtain neural progenitor cells (NPCs) as neurospheres, the cell aggregates were mechanically dissociated and cultured in DMEM/F12 containing $2 \%$ B27 supplement (Life Technologies), $20 \mathrm{ng} / \mathrm{ml}$ rh-basic fibroblast growth factor (bFGF; PeproTech, Rocky Hill NJ, USA), $20 \mathrm{ng} / \mathrm{ml}$ rh-epidermal growth factor (EGF; PeproTech), $10 \mathrm{ng} / \mathrm{ml}$ rh-leukemia inhibitory factor (LIF; Millipore, Billerica, MA, USA), and $5 \mu \mathrm{g} / \mathrm{ml}$ heparin (Sigma-Aldrich). The neurospheres were passaged before the center of the spheres darkened, typically every 12-14 days as described previously [17].

\section{Differentiation of the iPSC-derived neurospheres}

At approximately passage six, the neurospheres were plated onto GFR Matrigel (BD)-coated dishes in neurobasal medium (Life Technologies) containing $2 \%$ B27 supplement (Life Technologies) and $2 \mathrm{mM}$ L-glutamine (Life Technologies) (differentiation medium). For the PiggyBac vector transfection experiments, iPSC-derived neurospheres were used at stages beyond six passages when the regional identities could be caudalized and 
Table 1 Donor information and radiological findings

\begin{tabular}{|c|c|c|c|c|c|c|c|c|c|c|c|}
\hline Patient & Gender & $\begin{array}{l}\text { Age } \\
\text { (years) }\end{array}$ & Diagnosis & $\begin{array}{l}\text { LIS grading } \\
{[35]}\end{array}$ & Cerebral cortex & $\begin{array}{l}\text { Corpus } \\
\text { callosum }\end{array}$ & Cerebellum & $\begin{array}{l}\text { Basal } \\
\text { ganglia }\end{array}$ & Brain stem & Clinical information & $\begin{array}{l}\text { Same mutation reported } \\
\text { previously }\end{array}$ \\
\hline A & Male & 0 & $\begin{array}{l}\text { Microlissencephaly } \\
\text { with cerebellar } \\
\text { hypoplasia }\end{array}$ & Grade 1 & Lissencephaly & Agenesis & Hypoplastic & Hypoplastic & Hypoplastic & $\begin{array}{l}\text { Brain stem dysfunction, seizure, and } \\
\text { developmental delay. Died at } 2 \text { years } \\
\text { old. }\end{array}$ & Kumar RA et al. [5] \\
\hline B & Male & 2 & Lissencephaly & Grade 4 & $\begin{array}{l}\text { Agyria-pachygyria } \\
\text { (posterior }> \\
\text { anterior gradient) }\end{array}$ & $\begin{array}{l}\text { Present, } \\
\text { abnormal }\end{array}$ & $\begin{array}{l}\text { Not } \\
\text { hypoplastic }\end{array}$ & Hypoplastic & $\begin{array}{l}\text { Not } \\
\text { hypoplastic }\end{array}$ & $\begin{array}{l}\text { Intellectual delay and intractable } \\
\text { seizures. Word speech at } 3 \text { years old. } \\
\text { Has survived for } 4 \text { years now. }\end{array}$ & Keays DA et al. [4] \\
\hline
\end{tabular}


stably propagated. For differentiation in dissociated culture, the neurospheres were dissociated using TrypLE Select (Life technologies) for $5 \mathrm{~min}$ at $37{ }^{\circ} \mathrm{C}$, and the dissociated iPSC-NPCs were seeded onto GFR-Matrigel (BD)-coated 96-well plates and 6-well plates in differentiation media.

\section{Quantitative RT-PCR}

RNA was extracted from the aggregates and neurospheres using an RNeasy Mini Kit (QIAGEN) and DNase set (QIAGEN), and cDNAs were synthesized using PrimeStarRT Reagent Kits (Takara Bio, Shiga, Japan). Quantitative PCR was performed using a PowerSYBR Green PCR Mix (Life Technologies) and an ABI7300 Real-time PCR system (Life Technologies) using gene-specific primer pairs (Additional file 1: Table S1). The expression (Ct values) of each gene was normalized to that of an internal control, and the normalized expression was compared using the $\Delta \Delta \mathrm{Ct}$ method as previously described [18]. As a human telencephalic control, 201B7 iPSC-derived telencephalic SFEB (serumfree floating-culture of embryoid body-like) aggregates at day 25 [19] were used. GDC90 human glial cell lines were used as a human glial cell control [20].

\section{Visualization of the neurite extension of neurons derived from iPSCs of lissencephaly patients using the glia-supported neurite extension method}

We used the human glial cell line GDC90 as a scaffold [20] to support the robust differentiation and neurite extension of the iPSC-derived neurons. To distinguish glial cell lines and iPSC-derived neural progenitor cells (iPSC-NPCs) and to illustrate the morphology of the neurites migrating from each iPSC-NPC-derived young neuron, the CAG promoter-driven [21] PiggyBac vector pPB-CAG-EGFP-neo [20] was nucleofected into the iPSC-derived neurospheres using Amaxa Nucleofector I (Lonza) as previously described [20]. After positive selection using Geneticin at a concentration of $200 \mu \mathrm{g} / \mathrm{ml}$ (Life Technologies), the enhanced green fluorescent protein (EGFP)-labeled iPSC-NPCs were used for further analyses.

To measure the neurites migrating from the neurospheres, iPSC-NPCs $\left(5 \times 10^{3}\right.$ cells or $2.5 \times 10^{3}$ cells for time-lapse imaging) and GDC90s $\left(5 \times 10^{3}\right.$ cells or $7.5 \times$ $10^{3}$ cells for time-lapse imaging) were seeded and reaggregated in low-attachment 96-well plates with spindleshaped bottoms (Sumitomo Bakelite). After $24 \mathrm{~h}$, the re-aggregated spheres were plated onto GFR-Matrigelcoated glass-bottom dishes in differentiation medium. In some experiments requiring the visualization of the glial cell line, tdTomato-labeled GDC90s [20] were used.

To quantify the behavior of the iPSC-NPCs derived from lissencephaly patients, the length of the EGFPfluorescent neurites extending from the soma of the
iPSC-NPCs existing at the edge of the fluorescent clusters was measured using ImageJ software on day 5 . Time-lapse images during the differentiation and migration of the control and lissencephaly iPSC-derived neurons were acquired using an IncuCyte ZOOM HD (Essen BioSciences, Ann Arbor, MI, USA) every $4 \mathrm{~h}$. The video files were exported in AVI format at six frames per second.

\section{Immunocytochemistry and immunohistochemistry}

Undifferentiated iPSCs, forebrain-committed neurons, and neurospheres were fixed in $4 \%$ paraformaldehyde and stained using primary and secondary antibodies as described below. The samples were examined using a confocal laser-scanning microscope (LSM510, Carl Zeiss, Hallbergmoos, Germany). The following primary antibodies were used (host, dilution, manufacturer): neuronal class III $\beta$-tubulin (TUJ1; mouse, 1:500, Covance, Princeton, NJ), brain lipid-binding protein (BLBP; rabbit, 1:500, Millipore), GFAP (rabbit, 1:80, Sigma-Aldrich), SOX1 (mouse, 1:500, BD Pharmingen), NESTIN (rabbit, 1:500 [22] and mouse, 1:500, Millipore), GFP (mouse, 1:500, Clontech, Palo Alto, CA, USA), doublecortin (DCX; rabbit, 1:500, Cell Signaling), OCT-4 (mouse, 1:200, BD), NANOG (rabbit, 1:200, ReproCELL), TRA-1-60 (mouse, 1:200, Chemicon), SSEA-3 (rat, 1:200, Chemicon), SSEA-4 (mouse, 1:200, Chemicon), and TRA-1-81 (mouse, 1:200, Chemicon). The following secondary antibodies were used: goat anti-mouse IgG Alexa Fluor 488 (1:1000, Molecular Probes), goat anti-rabbit IgG Alexa Fluor 568 (1:1000, Molecular Probes), goat antirabbit IgG Alexa Fluor 488 (1:1000, Molecular Probes), goat anti-mouse IgG Alexa Fluor 648 (1:1000, Molecular Probes), goat anti-mouse IgM Alexa Fluor 568 (1:1000, Molecular Probes), goat anti-rat IgM Alexa Fluor 488 (1:1000, Molecular Probes), and goat anti-rabbit IgG Alexa Fluor 648 (1:1000, Molecular Probes).

\section{Results}

Generation of iPSCs derived from lissencephaly patients and control iPSCs

As a first step in generating iPSCs from patients with lissencephaly, gene-transferred UCCs and PBMCs were re-seeded onto the feeder cells of mouse embryonic fibroblasts on day 7 by using nucleofection, and both UCCs and PBMCs were reprogrammed 3 weeks after replating. In the established patient-derived iPSC lines, the colonies appeared like those of human embryonic stem cells (Fig. 2a). TUBA1A sequencing showed the heterozygous mutated sequence c. $986 \mathrm{~A}>\mathrm{G}$ or c.790C $>\mathrm{T}$ (Fig. 2b), and immunocytochemical analyses confirmed the expression of pluripotent marker genes (Fig. 2c). Karyotype analyses showed that these lines maintained a normal karyotype (Fig. 2d). We also established iPSCs 


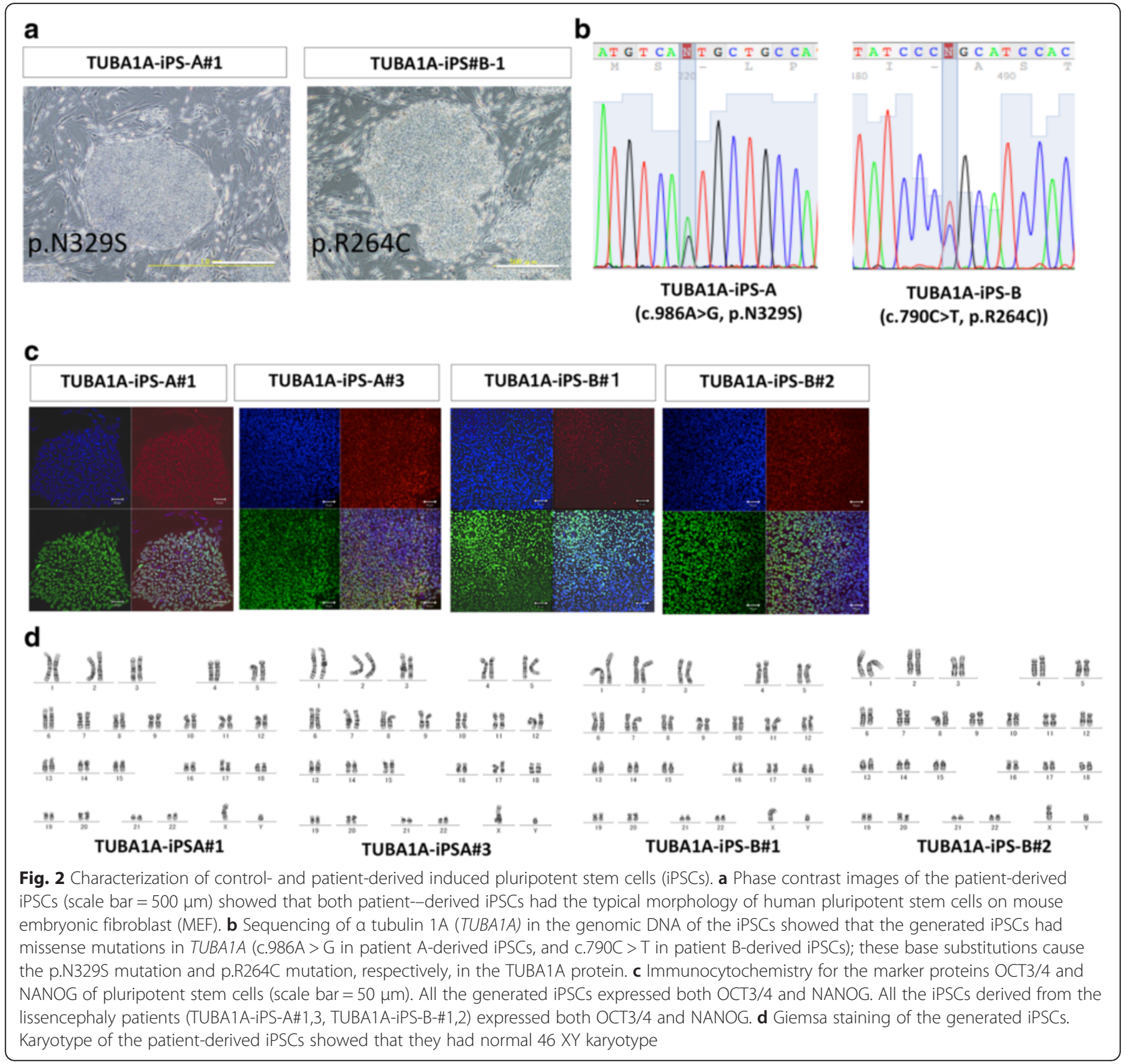

(ONH-UCCiPS1\#1 from UCC from a healthy volunteer using the same procedure as described above (Additional file 2: Figure S1A).

Quantitative real-time PCR showed that these lines expressed pluripotent cell markers similar to those expressed in the human embryonic stem cells KhES1 (Additional file 2: Figure S1B). These results showed that the newly generated iPSCs differentiated into three germ layers, suggesting that they were pluripotent. PCR analysis of the genomic DNA confirmed that the episomal vector was absent in these iPSCs (Additional file 2: Figure S1C). A teratoma formation assay using subcutaneous transplantation into severely immunodeficient NOG mice was conducted to test for differentiation (Additional file 2:
Figure S1D). Collectively, these findings confirmed that the established human iPSC clones in the present study met the general criteria for human iPSCs.

\section{Generation of iPSC-NPCs using dual SMAD inhibition and} neurosphere culture

After dual SMAD inhibition, the EBs containing neural progenitor cells were grown for 14 days. Following EB dissociation, proliferative iPSC-NPCs were generated using neurosphere culture with serum-free medium containing bFGF-2, EGF, and LIF (Fig. 3a). Quantitative RT-PCR analysis for TUBA1A, $1 B$, and $1 C$ showed that only $1 \mathrm{~A}$ showed remarkable activation during neural induction, suggesting that phenotypic analyses should be performed 


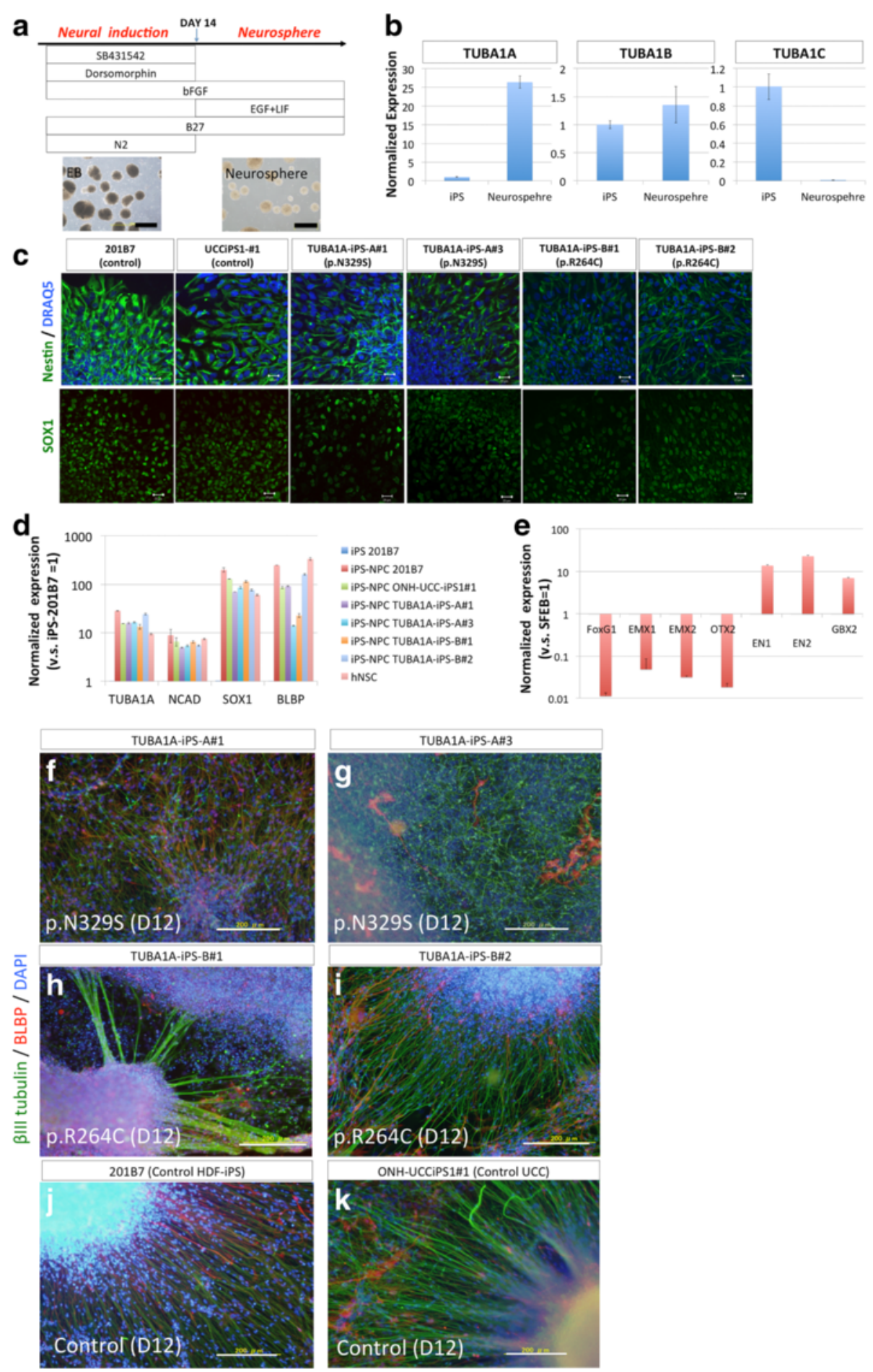

Fig. 3 (See legend on next page.) 


\section{(See figure on previous page.)}

Fig. 3 Generation of neurospheres from the induced pluripotent stem cells (iPSCs). a Schema of neural induction and neurosphere generation. Neural induction using dual SMAD inhibition and subsequent neurosphere cultures were conducted to obtain the neurospheres. After 2 weeks of neural induction, the embryoid bodies (EBs) were dissociated and cultured in a medium containing bFGF, EGF, and LIF (scale bar $=50 \mu \mathrm{m})$. Representative photographs of EBs and neurospheres were derived from Patient A. b Quantitative RT-PCR for each TUBA1 isoform. Through neural induction, only TUBATA expression was greatly increased. Changes in the expression of each TUBA1 isoform were different (relative to the expression in the undifferentiated iPSCs (201B7), fold change \pm SD, $n=3$, technical duplicates). c Immunostaining of each neurosphere (within $12 \mathrm{~h}$ after plating in neurosphere medium). Ubiquitous staining for the NPC markers NESTIN and SOX1 (scale bar = 50 $\mu$ m) was observed in each cell. d Quantitative RT-PCR showed that the neurospheres expressed the neural marker genes N-cadherin (CDH2) and a tubulin 1A (TUBATA) and the progenitor cell marker SOX1, all of which are expressed in the human neural stem cell (hNSC) line. The radial glial marker brain lipid-binding protein (BLBP) was also expressed (relative to the expression in the undifferentiated iPS, fold change \pm SD, $n=3$, technical duplicates). e Quantitative RT-PCR showed that the neurospheres had a caudalized regional identity characterized by high gastrulation brain homeobox 2 (GBX2) expression and low Forkhead box protein G1 (FOXG1) expression (relative to the expression of SFEB used as human forebrain control, fold change $\pm S D, n=3$, technical duplicates). $\mathbf{f}-\mathbf{k}$ Spontaneous differentiation of the neurospheres. In 12 days, the $\beta$ III tubulin-positive neurites and few BLBP-positive radial glial-like fibers had migrated radially from the neurospheres (scale bar $=200 \mu \mathrm{m}$ ). Poorly ordered neurites and BLBP-positive radial glial-like fibers were observed in the TUBA1A-iPS-A-derived neurospheres ( $\mathbf{f}-\mathbf{g})$. Radially extending neurites and BLBP-positive radial glial-like fibers were observed in the TUBA1A-iPS-B-derived neurospheres $(\mathrm{H}-\mathrm{I})$ and control iPSC-derived neurospheres $(\mathbf{j}-\mathbf{k})$

in the neurospheres and not in the undifferentiated iPSCs (Fig. 3b). The cells in the neurospheres homogeneously expressed the NPC markers SOX1 and NESTIN (Fig. 3c), as confirmed by immunocytochemistry, which indicated that these cells contained NPCs with high purity. Furthermore, quantitative RT-PCR analyses showed that the neural marker $\mathrm{N}$-Cadherin and the NPC markers SOX1 and $B L B P$ were highly expressed in each neurosphere (Fig. 3d). Together, these data indicate that iPSC-NPCs could be generated by dual SMAD inhibition and could be proliferated as neurospheres.

TUBA1A mutation-associated lissencephaly is characterized by cerebellar hypoplasia and often involves the hypoplasia of the brain stem, including the midbrain and pons. Thus, it is important to determine the regional identity of the neurospheres generated using this method. We therefore performed expression analyses for the region-specific markers.

Interestingly, these propagated neurospheres expressed forkhead box G1 (FOXG1; a forebrain marker) at a lower level and gastrulation brain homeobox 2 (GBX2; a hindbrain marker) a higher level compared with the miniature brain called "SFEB", which was used as a human telencephalic control and was generated using previously described methods [19]. This result indicated that these neurospheres belonged to the caudal region, which includes the hindbrain (Fig. 3e).

Soon after differentiation, these neurospheres contained glial, neuronal, and neural progenitor cells, all of which play important roles in fetal CNS development. When the neurospheres were plated, $\beta I I I$ tubulin-positive neuronal cells and several BLBP-positive glial fibers spread toward the outside. BLBP-positive fibers are highly polarized and consist of long processes that extend out of the neurospheres, similar to what was observed for radial glial cells during CNS development (Fig. 3f-k).

At first, we simply compared the morphology of the differentiating neurospheres on Matrigel-coated dishes during early passages (6-8 passages) when the BLBPpositive glial fibers were often observed. From these neurospheres, many neurites extended together with BLBP-positive glial fibers after 12 days (Fig. 3f-k).

Interestingly, the neurites and glial fibers derived from the iPSC-NPCs of the lissencephaly patient A (with the p.N329S TUBA1A mutation) did not extend radially and displayed a shorter morphology (Fig. 3f-g). In contrast, the neuronal cells and glial fibers derived from the iPSCNPCs from patient B carrying the p.R264C TUBA1A mutation projected radially (Fig. $3 \mathrm{~h}-\mathrm{i}$ ). We believe that these results might be associated with the patient's pathology. However, quantitative analyses of neuronal morphology was technically challenging due to significant increases in cell density during the 12 days of in vitro differentiation required. Furthermore, undifferentiated NPCs persisted in the center of the neurospheres and continued to produce newborn neurons, which would further complicate quantitative analyses of neuronal morphology such as Sholl analysis.

The number of BLBP-positive glial cells in the neurospheres was low and varied between neurospheres and cell lines, which is consistent with the results of quantitative RT-PCR for BLBP (Fig. 3d). To promote the differentiation of NPCs [23] and to reduce variation, establishing cultures with comparable glial content is critical. However, propagation using conditions conducive for neurospheres tends to make the iPSC-NPCs neurogenic rather than gliogenic [24, 25] (Additional file 3: Figure S2), and the precise control of the glial differentiation propensity of the iPSCs remains technically difficult. Therefore, we supplemented the cultures with human glial cells in order to induce robust and rapid differentiation of the iPSC-NPCs.

\section{Glia-supported neurite extension with fluorescently labeled iPSC-NPCs in neurospheres}

Genetic labeling with a PiggyBac transposon system was used to establish stable fluorescent iPSC-NPCs to 
distinguish them from glial cells (Fig. 4a). The iPSC-NPCs were easily propagated by neurosphere culture, enabling the transfection, selection, and propagation of fluorescent cells. The iPSC-NPCs that were co-cultured with the GDC90 cells rapidly polarized and extended their neurites radially compared with those that were grown in the absence of glial cells (Fig. 4b-d and Additional files 4 and 5: Movies S1 and S2). Immunocytochemistry showed that most of the EGFP-positive (iPSC-NPC-derived) neurites expressed DCX, which is a marker for young migrating neurons (Fig. 4e). Time-lapse imaging showed that the EGFP-positive neurites projected radially, and that some of their somas were pulled up from the core of the neurosphere in translocation-like movements in 12 days (Additional files 5 and 6: Movies S2 and S3).

\section{Analyses of extending neurites using glia-supported neurite extension methods}

The behaviors of the patient-derived and control iPSCNPCs were evaluated using the glia-supported neurite extension method. The measurement of neurite extension after 5 days showed that neurite extension in iPSC-NPCs derived from the patient with the p.N329S TUBA1A mutation was severely inhibited (Fig. $4 \mathrm{f}-\mathrm{g}$ and $\mathrm{l}$, Additional files 7 and 8: Movies S4, S5). These data suggest that neuronal cells that differentiated from the iPSC-NPCs with the p.N329S TUBA1A mutation had an immature morphology compared with those derived from the control iPSC-NPCs. We believe that this phenotype corresponded to the pathology of the developing brain because neuronal cells initially polarize from NPCs and migrate toward the cortical plate using various types of neurites, including the leading process and basal process.

This observation was also supported by the results of our experiment using human fetal forebrain-derived NPCs, in which we introduced the CMV early enhancer/ chicken beta actin (CAG) promoter-driven p.N329S mutant TUBA1A using the PiggyBac transposon based vector system. The overexpression of p.N329S mutant TUBA1A significantly reduced neurite extension of human NPCs (Additional file 9: Figure S3, Additional file 10: Supplementary method), suggesting that lissencephaly related to the p.N329S TUBA1A mutation was caused by a dominant-negative effect. Together with the results of our experiments described above, we concluded that we had successfully modeled one aspect of the pathology of lissencephaly in vitro using patient-derived iPSC-NPCs with the p.N329S TUBA1A mutation.

In contrast, we did not identify any phenotypic difference between the iPSC-NPCs with a p.R264C TUBA1A mutation and those derived from the controls (Fig. $4 \mathrm{~h}-\mathrm{k}$, Additional file 11: Movie S6). One reason for this result may be the disease severity of the patient. The corpus callosum was missing in Patient A (with the N329S mutation), (Fig. 1a-b, Fig. 4m) whereas it is detectable in Patient B (with the p.R264C mutation), as determined from the MR images (Fig. 1c-d, Fig. 4n), suggesting that neurite extension was not as severely affected in Patient B as in Patient A. This finding is consistent with our present in vitro results obtained by measuring neurite extension (Fig. 4l). Importantly, this result was also consistent with the gradient in the pathology along the cephalic-caudal axis, as observed in the patients. The brain stem and thalamus, which have a regional identity similar to that of our iPSC-NPCs, did not appear hypoplastic in the MR images obtained from Patient B (Fig. 1d, Table 1).

Overall, the p.N329S TUBA1A mutation observed in Patient A showed reduced neurite extension that was caused by mutated tubulin in a dominant-negative manner. In contrast, the neurons generated from the iPSCs from Patient B with the p.R264C TUBA1A mutation showed no overt defects in neurite extension. This difference was consistent with the radiological pathology of the TUBA1A lissencephaly patients.

\section{Discussion}

In the present study, we established iPSCs from an easily accessible cell source from patients with lissencephaly and successfully characterized the abnormalities of the iPSC-derived neurons in vitro. Recent progress in cell reprogramming methods enabled us to generate iPSCs from a variety of cell sources $[9,11,26]$. For research on human congenital brain malformations, the utility of patient-derived iPSCs overcomes the hurdles that arise owing to the lack of availability of patient-derived cells in extremely rare diseases. Furthermore, we successfully converted these cells into expandable and easy-to-handle iPSC-NPCs using neurosphere culture technology; these resources will be useful in human congenital malformation research in the future.

Lissencephaly is often caused by mutations in microtubule-associated genes [27]. Polarized neuronal cells depend on the microtubule pathway, whereas non-polarized cells depend primarily on the driving force of actin-myosin contraction. To model lissencephaly pathology in the iPSC-NPCs, the dynamic behavior of the polarized neuronal cells must be visualized.

Spontaneously differentiating neurospheres showed extensions of small proportions of BLBP-positive radial fibers and aligned $\beta \mathrm{III}$ tubulin-positive neurons. In our simple assay, randomly oriented neurons migrated from the neurospheres generated from the iPSCs with the p.N392S TUBA1A mutation, which might partially reflect the in vivo pathology of the patients with TUBA1A mutation-associated lissencephaly. To reduce the variation associated with the glial content of each neurosphere, we used a human glial cell line for robust and rapid neuronal differentiation. Moreover, we could observe the 







\begin{abstract}
(See figure on previous page.)
Fig. 4 Glia-supported neurite extension method for fluorescently labeled induced pluripotent stem cell (iPSC)-derived neurospheres. a Schema of the glia-supported neurite extension method with fluorescently labeled iPSC-derived neurospheres. $\mathbf{b}, \mathbf{c}$ Effect of the glia-supported neurite extension method on the fluorescently labeled iPSC-derived neurospheres. After 3 days, the non-polarized cells spread in a disordered manner without a glial cell line; however, using glia-supported neurite extension methods (with GDC90), the differentiating neurites were found to migrate from the neurospheres in a radial and orderly manner (scale bar $=300 \mu \mathrm{m}$ ). Rapid and straightforward extension of the neurites was easily observed using strong EGFP fluorescence. $\mathbf{d}$ Fluorescence and phase contrast merged images of the glia-supported neurite extension method using EGFP-labeled iPSC-derived neurospheres and tdTomato-labeled human glial GDC90 cells (scale bar $=200 \mu \mathrm{m}$ ). e Immunostaining for the young neuron marker doublecortin (DCX) showed that the neurites extending on the tdTomato-positive human glial cells were also stained with EGFP (scale bar $=50 \mu \mathrm{m}$ ). $\mathbf{f}-\mathbf{k}$ EGFP fluorescence of the extending neurites observed using the glia-supported neurite extension method on day 5 after plating on the dish. Patient A (p.N329S)-derived neural progenitor cells (NPCs) showed poor neurite extension (f--g) compared to the Patient B (p.R264C)-derived (h-i) or healthy control iPSC-derived NPCs $(\mathbf{j}-\mathbf{k})($ scale bar $=200 \mu \mathrm{m})$. I Length of EGFP-positive neurites extending from the neurospheres $(\mu \mathrm{m}$ : mean \pm SEM). Boxplot graph illustrating median, first quartile, and third quartile with each outliner plotted. $(* * p<0.01$, one-way ANOVA followed by Dunnett's test. $n=269,277,374,369,338$, and 213 in A\#1, A\#3, B\#1, B\#2, 201 B7 (control), and UCCiPS1\#1, respectively. Statistical analyses were performed using 201 B7 as the control. When using ONH-UCCiPS1 independently as a control, the same results were also confirmed.). $\mathbf{m}, \mathbf{n}$ Magnified images of coronal scanned MR images showing the morphological differences in the corpus callosum between Patient A (T2-weighted) and Patient B (T1-weighted). The corpus callosum was not present in the MR images (M) of Patient A (with the p.N329S mutation), suggesting that the interhemispheric projection was hypoplastic, unlike that in Patient B (with the p.R264C mutation). Poor neurite extension of patient-derived iPSCs was considered to correspond to the patient's phenotype and severity of the disease
\end{abstract}

characteristics of the patient-derived neurons themselves without the effects of the endogenous glial cells, which might be susceptible to the effects of mutated TUBA1A or the secondary effects of abnormal neuron-mediated morphological changes that may spoil endogenous glial scaffolds [28]. We also introduced a fluorescent protein into the iPSC-NPCs to observe the behavior of the individual cells using the PiggyBac transposon system. Interestingly, the control iPSC-NPCs in this glial cell scaffold rapidly differentiated into polarized neuronal cells with radially extending neurites. Using this system, we found that the neurite extension from the iPSC-NPCs with the p.N329S TUBA1A mutation was inhibited, whereas the iPSC-NPCs carrying the p.R264C mutation showed no apparent abnormality, indicating agenesis of the corpus callosum in Patient A with the N329S mutation. This observation suggested that patient-derived iPSC-NPCs could recapitulate the neurite extension process in the patient brain. Furthermore, neuronal cells with short neurites were also considered immature. Although we have no histological data for these patients, these neurons were considered similar to those of the patient brain because a differentiation defect manifested by immature neurons and persistent radial glia was reported previously in the TUBA1A lissencephaly patient brain [27]. Based on our data from the iPSC-derived NPCs, we concluded that the pathology of p.N329S TUBA1A lissencephaly might start from the steps of differentiation or polarization from neural progenitor cells, leading to a migration defect in young neurons and lamination defects in the cerebral cortex. We suspect that p.N329S TUBA1A destabilizes tubulin heterodimer formation by decreasing the number of hydrogen bonds between alpha- and beta-tubulin in the neuronal cells that express abundant TUBA1A for neurite extension [5]. This hypothesis is consistent with the dominant-negative action of p.N329S mutant
TUBA1A on neurite extension, determined from the overexpression experiments (Additional file 9: Figure S3). However, considering its effects on the phenotype, the effect of overexpressing the mutant gene would be artificial. Therefore, to investigate the role of N329S, R264C, and other mutations in the disease-related phenotype, further biochemical characterizations, in patient-derived iPSCs and isogenic iPSCs introduced with these mutations by genome editing, is required in our future experiments.

As shown above, we successfully recapitulated one aspect of the pathology of lissencephaly, but the important question of whether neurons derived from a lissencephaly patient can migrate normally has not been answered. The disorganized lamination of the cerebral cortex in lissencephaly patients cannot be easily explained only by the delayed differentiation of neural progenitor cells or the delay in polarization before neuronal migration. To answer this question, further experiments involving the direct observation of migrating human iPSC-derived cortical neurons are needed. We have already generated a miniature brain from iPSCs derived from a patient with severe lissencephaly. However, unexpectedly, we found young cortical neurons located outside the germinal layer (data not shown). The exact mechanism of cortical layer formation in vitro needs to be determined before interpreting this result.

We observed translocation-like movements in this study, which led us to believe that our co-culture system might be useful in the neuronal migration of iPSCderived telencephalic neurons.

Consistent with the MR images of the lissencephaly patient $\mathrm{B}$, who had a less severe phenotype, iPSC-NPCs with the p.R264C TUBA1A mutation showed an almost normal neurite extension, although it is possible that the sensitivity of our assay system was not sufficient to detect the slight change in the phenotype. In contrast to 
p.N329S TUBA1A, p.R264C TUBA1A was reported to decrease the production of TUBA1A by disturbing protein folding, which might be the reason for the difference in disease severity; that is, the shortage of TUBA1A caused by the p.R264C TUBA1A mutation could be compensated for by other interchangeable $\alpha$ tubulin isoforms via region-specific transcriptional regulation [29-31]. Regional phenotypic differences in TUBA1A mutation-associated lissencephaly also might be partially explained by differences in the transcriptional regulation of TUBA1A throughout the brain. Further investigation into TUBA1A regulation is needed to understand the detailed mechanism of TUBA1A-associated lissencephaly.

Finally, we hypothesized that lissencephaly may provide the key to understanding other neurodevelopmental disorders. For example, the pathological analysis of postmortem brain tissue from a patient with autism revealed a partial migration defect [32]. Moreover, the $M e C P 2$ gene, which is associated with syndromic autism and Rett syndrome, is thought to modulate TUBA1A expression [33].

Thus, we believe that an experimental system using iPSC-derived neurospheres from cases of congenital brain malformation would be useful for elucidating the mechanism of the diseases and the development of the human brain.

\section{Conclusion}

We established iPSCs derived from two TUBA1Aassociated lissencephaly patients and successfully modeled one aspect of the pathogenesis of lissencephaly in vitro using iPSC-NPCs and iPSC-derived neurons. The disease modeling using iPSCs from patients with brain malformation diseases helped us understand the mechanism underlying rare diseases and human brain development.

\section{Additional files}

Additional file 1: Table S1. Primer sequences used in this study. * These primers were used in quantitative PCR for pluripotent stem cell markers. (DOCX $19 \mathrm{~kb}$ )

Additional file 2: Figure S1. (A) Characterization of umbilical cord stromal cell-derived control iPSCS (ONH-UCCiPS1\#1). ONH-UCCiPS1\#1 is morphologically similar to human embryonic stem cells on a mouse embryonic fibroblast feeder. Immunocytochemistry showed that they expressed the pluripotent stem cell markers OCT3/4 and NANOG ubiquitously. (B) Quantitative RT-PCR for marker genes of pluripotent stem cells (Relative to the expression in control human embryonic stem cells KhES1, fold change $\pm \mathrm{SD}, n=3$, technical duplicates) showed that the generated iPSCs expressed the marker genes. (C) Conventional PCR for the detection of persistence of the episomal vector confirmed no major persistence of plasmid vectors (EP4 stands for the primer set designed using detection of the episomal vector-specific sequence). (D) Teratoma formation assay using subcutaneous transplantation in NOD/Shi-scid IL2Rg null (NOG) mice (scale bar $=100 \mu \mathrm{m}$ ) showed that the patient-derived iPSCs could differentiate into three germ layers. (TIF $19759 \mathrm{~kb}$ )

Additional file 3: Figure S2. (A-C) Immunocytochemistry for the immature neuronal marker BIII tubulin and glial cell marker GFAP. Most of the dissociated iPSC-NPCs were differentiated into neurons in serum-free differentiation medium within 2 weeks. (D) Quantification of data from immunocytochemical analyses showed that the dissociated iPSC-NPCs were differentiated predominantly ( $>90 \%)$ into Blll tubulin neurons in 2 weeks. GFAP-positive cells were very rarely $(<1 \%)$ observed in our differentiation condition (mean \pm SD, $n=3$ technical duplicates of each cell line, control; 201B7 and UCCiPS1\#1, p.N329S; TUBA1A-iPS-A\#1, A\#3, p.R264C; TUBA1A-iPS-B\#1, B\#2). No significant differences in the neurogenicity and gliogenicity were observed between these cells (Welch's $t$ test). (E) Quantitative RT-PCR showed that the expression of the glial marker GFAP in the differentiated iPSC-NPCs was extremely low (mean \pm SD, $n=3$ technical duplicates of each cell line, control; 201B7 and UCCiPS1\#1, p.N329S; TUBA1A-iPS-A\#1, A\#3, p.R264C; TUBA1A-iPS-B\#1, B\#2). This result was consistent with the data obtained from the immunocytochemical analyses shown in Additional file 3: Figure S2A. In addition, there were no significant differences in GFAP expression between these cell lines (Welch's $t$ test). (F) Quantitative PCR showed that the immature neuronal marker TUBB3 in the differentiated iPSC-NPCs was expressed in all the cell lines, and that the differences were not statistically significant (Welch's $t$ test, mean $\pm S D, n=3$ technical duplicates of each cell line, control; 201B7 and UCCiPS1\#1, p.N329S; TUBA1A-iPS-A\#1, A\#3, p.R264C; TUBA1A-PPS-B\#1, B\#2). (TIF $19759 \mathrm{~kb}$ )

Additional file 4: Movie S1. Fluorescent neurosphere (201B7) differentiation without glial cells. (AVI $25686 \mathrm{~kb}$ )

Additional file 5: Movie S2. Fluorescent neurosphere (201B7) differentiation with glial cells. (AVI $30161 \mathrm{~kb}$ )

Additional file 6: Movie S3. Translocation-like movement in differentiating fluorescent neurospheres (201B7) at high magnification. (AVI $2472 \mathrm{~kb}$ )

Additional file 7: Movie S4. Neurite extension (201B7) with the gliasupported neurites extension method. (AVI $1390 \mathrm{~kb}$ )

Additional file 8: Movie S5. Neurite extension (iPS-A) with the gliasupported neurites extension method. (AVI $891 \mathrm{~kb}$ )

Additional file 9: Figure S3. Differentiating PPBCAG-TUBA1A-IRESAcGFP-transfected human NPCs. Measurements of AcGFP-positive neurites from each cell ( $\mu \mathrm{m}$ : mean $\pm \mathrm{SEM}, n=50$, one-way ANOVA followed by Dunnett's test, ${ }^{* *} p<0.01$ ). Overexpression of mutant TUBA1A (p.N329S) interfered with neurite extension in the human NSCs (scale bar $=200 \mu \mathrm{m}$ ). (TIF $19759 \mathrm{~kb}$ )

Additional file 10: Supplementary method. (DOCX $72 \mathrm{~kb}$ )

Additional file 11: Movie S6. Neurite extension (iPS-B) with the gliasupported neurites extension method. (AVI $1492 \mathrm{~kb}$ )

Abbreviation

NPC, neural progenitor cell; iPSC, induced pluripotent stem cells; SFEB, serum-free embyoid body like aggregates; TUBA1A, alpha tubulin 1A; TUBB3, beta III tubulin; GFAP, glial fibrillary acidic protein; BLBP, brain lipid-binding protein; EGFP, enhanced green fluorescent protein; FOXG1, Forkhead box protein $\mathrm{G} 1$; GBX2, gastrulation brain homeobox 2

\section{Acknowledgements}

We thank Dr. Chiaki Ban, Ms. Chika Teramoto and Ms. Ai Takada for the collection of human umbilical cord tissues, and all the staff members of our laboratories and all the collaborators in the Fetal Brain Malformation (FBM) Network in Japan. Especially, we fully appreciate Hayato Fukusumi for the technical assistance in our revised manuscript. We also give our thanks for the kind gift of episomal vectors that were used in iPSC generation from Dr. Keisuke Okita and Prof. Shinya Yamanaka. This study is supported by the Research on Intractable Diseases and the Research on Applying Health Technology, Health and Labour Sciences Research Grants from the Ministry of Health, Labour and Welfare of Japan to M.Y., and the Program for Intractable Disease Research Utilizing Disease-specific iPS Cells funded by 
the Japan Science and Technology Agency (JST)/Japan Agency for Medical Research and Development (A-MED) to H.O..

\section{Funding}

This research is supported by Health and Labor Sciences Research Grants for research of intractable disease to M.Y. (Award Number: 2009-ID-028, 2010-ID131, 2011-ID-013) and Program for Intractable Disease Research Utilizing Disease-specific iPS Cells funded by the Japan Science and Technology Agency (JST)/Japan Agency for Medical Research and Development (A-MED) to H.O.(Award Number: 0609003h)

\section{Authors' contributions}

This research was designed by $\mathrm{YB}, \mathrm{YK}, \mathrm{MY}, \mathrm{WA}$, and $\mathrm{HO}$ and was performed in the laboratory of YK and YM. The experiments were performed by YB, TS, DK and MS. The teratoma formation assay was performed by $\mathrm{YH}$ and HS in CIEA as described in the material and methods section. The interpretation of clinical imaging data and clinical information and the sampling of patient-derived materials were performed by MK, RKP, YT, JT, and HU. The data were analyzed by $Y B, T S, Y K$, and $H O$. Detailed protocols for the neural induction of pluripotent stem cells were developed and provided by DG and FDM. The conception of this research was made by $Y M, Y K$, and $H O$. This manuscript was written by $Y B$, $\mathrm{YK}, \mathrm{MY}$, and $\mathrm{HO}$. All the authors approved the final version of the manuscript.

\section{Competing interests}

Dr. H. Okano is a paid scientific consultant to SanBio Co Ltd and Eisai Co., Ltd. Dr. Y. Kanemura received research funding from Kaneka Corp. The authors declare no conflicts of interest associated with this manuscript.

\section{Ethics approval and consent to participate and consent for publication} This study was conducted in accordance with the principles of the Helsinki Declaration, and the approval for the use of human tissues and cells and for genetic examination was obtained from the ethics committees of Osaka National Hospital and Keio University School of Medicine (No. 94, 110, 123, 146, and 150) with appropriate informed consent including participation and publication.

\section{Author details}

'Department of Physiology, Keio University School of Medicine, 35 Shinanomachi, Shinjuku-ku, Tokyo 160-8582, Japan. ${ }^{2}$ Division of Regenerative Medicine, Institute for Clinical Research, Osaka National Hospital, National Hospital Organization, 2-1-14 Hoenzaka, Chuo-ku, Osaka 540-0006, Japan. ${ }^{3}$ Division of Stem Cell Research, Institute for Clinical Research, Osaka National Hospital, National Hospital Organization, Osaka, Japan. ${ }^{4}$ Department of Pediatrics, Yamagata University Faculty of Medicine, 2-2-2 lida-Nishi, Yamagata-shi, Yamagata 990-9585, Japan. ${ }^{5}$ CRIFM Clinical Research Institute of Fetal Medicine Pooh Maternity Clinic, 7-1-24 Uehommachi, Tennoji, Osaka 543-0001, Japan. ${ }^{6}$ Department of Obstetrics and Gynecology, Okayama Medical Center, National Hospital Organization, 1711-1 Tamasu, Okayama, Japan. ${ }^{7}$ Department of Pediatrics, Tokyo Women's Medical University, Yachiyo Medical Center, 477-96 Owadashinden, Yachiyo-shi 276-8524, Japan. ${ }^{8}$ Department of Radiological Science, International University of Health and Welfare, Graduate School, 2-4-16, Momochihama, Sawara-ku, Fukuoka 814-0001, Japan. 'Laboratory Animal Research Department, Central Institute for Experimental Animals, 3-25-12 Tonomachi, Kawasaki-ku, Kawasaki, Kanagawa 210-0821, Japan. ${ }^{10}$ Program in Neuroscience and Mental Health, The Hospital for Sick Children, Toronto, ON M5G 0A7, Canada. ${ }^{11}$ McEwen Center for Regenerative Medicine, University Health Network, Toronto, ON M5G 0A7, Canada. ${ }^{12}$ Department of Molecular Genetics, University of Toronto, Toronto, ON M5G1X5, Canada. ${ }^{13}$ Department of Physiology, University of Toronto, Toronto, ON M5G 1X5, Canada. ${ }^{14}$ Department of Pediatric Neurosurgery, Takatsuki General Hospital, 1-3-13、Kosobe cho, Takatsuki 569-1192, Japan. ${ }^{15}$ Division of Molecular Medicine, Institute for Clinical Research, Osaka National Hospital, National Hospital Organization, Osaka, Japan. ${ }^{16}$ Department of Neurosurgery, Osaka National Hospital, National Hospital Organization, Osaka, Japan.

Received: 13 January 2016 Accepted: 24 April 2016 Published online: 19 July 2016

\section{References}

1. Rakic P. Evolution of the neocortex: a perspective from developmental biology. Nat Rev Neurosci. 2009;10:724-35.

2. Lui JH, Hansen DV, Kriegstein AR. Development and evolution of the human neocortex. Cell. 2011;146:18-36.

3. Florio $M$, Huttner WB. Neural progenitors, neurogenesis and the evolution of the neocortex. Development. 2014;41:2182-94.

4. Keays DA, Tian G, Poirier K, Huang GJ, Siebold C, Cleak J, Oliver PL, Fray M, Harvey RJ, Molnar Z, et al. Mutations in alpha-tubulin cause abnormal neuronal migration in mice and lissencephaly in humans. Cell. 2007;128: 45-57.

5. Kumar RA, Pilz DT, Babatz TD, Cushion TD, Harvey K, Topf M, Yates L, Robb S, Uyanik G, Mancini GM, et al. TUBA1A mutations cause wide spectrum lissencephaly (smooth brain) and suggest that multiple neuronal migration pathways converge on alpha tubulins. Hum Mol Genet. 2010;19:2817-27.

6. Poirier K, Keays DA, Francis F, Saillour Y, Bahi N, Manouvrier S, Fallet-Bianco C, Pasquier L, Toutain A, Tuy FP, et al. Large spectrum of lissencephaly and pachygyria phenotypes resulting from de novo missense mutations in tubulin alpha 1A (TUBA1A). Hum Mutat. 2007;28:1055-64.

7. Kanematsu D, Shofuda T, Yamamoto A, Ban C, Ueda T, Yamasaki M, Kanemura Y. Isolation and cellular properties of mesenchymal cells derived from the decidua of human term placenta. Differentiation. 2011;82:77-88.

8. Goto S, Kaneko T, Miyamoto Y, Eriguchi M, Kato A, Akeyama T, Fujimoto K, Tomonaga M, Egawa K. Combined immunocell therapy using activated lymphocytes and monocyte-derived dendritic cells for malignant melanoma. Anticancer Res. 2005;25:3741-6.

9. Takahashi K, Tanabe K, Ohnuki M, Narita M, Ichisaka T, Tomoda K, Yamanaka S. Induction of pluripotent stem cells from adult human fibroblasts by defined factors. Cell. 2007;131:861-72.

10. Okita K, Matsumura Y, Sato Y, Okada A, Morizane A, Okamoto S, Hong H, Nakagawa M, Tanabe K, Tezuka K, et al. A more efficient method to generate integration-free human iPS cells. Nat Methods. 2011:8:409-12.

11. Okita K, Yamakawa T, Matsumura Y, Sato Y, Amano N, Watanabe A, Goshima $\mathrm{N}$, Yamanaka S. An efficient nonviral method to generate integration-free human-induced pluripotent stem cells from cord blood and peripheral blood cells. Stem Cells. 2013;31:458-66.

12. Shofuda T, Kanematsu D, Fukusumi H, Yamamoto A, Bamba Y, Yoshitatsu S, Suemizu H, Nakamura M, Sugimoto Y, Furue M, Kohara A, Akamatsu W, Okada Y, Okano H, Yamasaki M, Kanemura Y. Human decidua-derived mesenchymal cells is a promising source for generation and cell banking of human induced pluripotent stem cells. Cell Med. 2013;4:125-47.

13. Ito M, Hiramatsu H, Kobayashi K, Suzue K, Kawahata M, Hioki K, Ueyama Y, Koyanagi Y, Sugamura K, Tsuji K, et al. NOD/SCID/gamma(c)(null) mouse: an excellent recipient mouse model for engraftment of human cells. Blood. 2002;100:3175-82.

14. Suemori H, Yasuchika K, Hasegawa K, Fujioka T, Tsuneyoshi N, Nakatsuji N. Efficient establishment of human embryonic stem cell lines and long-term maintenance with stable karyotype by enzymatic bulk passage. Biochem Biophys Res Commun. 2006;345:926-32.

15. Lowe J, Li H, Downing KH, Nogales E. Refined structure of alpha betatubulin at 3.5 A resolution. J Mol Biol. 2001;313:1045-57.

16. Wang J, Gallagher D, DeVito LM, Cancino Gl, Tsui D, He L, Keller GM, Frankland PW, Kaplan DR, Miller FD. Metformin activates an atypical PKCCBP pathway to promote neurogenesis and enhance spatial memory formation. Cell Stem Cell. 2012;11:23-35.

17. Kanemura Y, Mori H, Kobayashi S, Islam O, Kodama E, Yamamoto A, Nakanishi Y, Arita N, Yamasaki M, Okano H, et al. Evaluation of in vitro proliferative activity of human fetal neural stem/progenitor cells using indirect measurements of viable cells based on cellular metabolic activity. J Neurosci Res. 2002;69:869-79.

18. Shofuda T, Fukusumi H, Kanematsu D, Yamamoto A, Yamasaki M, Arita N, Kanemura Y. A method for efficiently generating neurospheres from human-induced pluripotent stem cells using microsphere arrays. Neuroreport. 2013;24:84-90.

19. Kadoshima T, Sakaguchi H, Nakano T, Soen M, Ando S, Eiraku M, Sasai Y Self-organization of axial polarity, inside-out layer pattern, and speciesspecific progenitor dynamics in human ES cell-derived neocortex. Proc Natl Acad Sci U S A. 2013;110:20284-9.

20. Bamba Y, Shofuda T, Kanematsu D, Nonaka M, Yamasaki M, Okano H, Kanemura Y. Differentiation, polarization, and migration of human induced pluripotent stem cell-derived neural progenitor cells co-cultured with a 
human glial cell line with radial glial-like characteristics. Biochem Biophys Res Commun. 2014;447:683-8.

21. Niwa H, Yamamura K, Miyazaki J. Efficient selection for high-expression transfectants with a novel eukaryotic vector. Gene. 1991;108:193-9.

22. Nakamura $Y$, Yamamoto M, Oda E, Yamamoto A, Kanemura Y, Hara M, Suzuki A, Yamasaki M, Okano H. Expression of tubulin beta II in neural stem/ progenitor cells and radial fibers during human fetal brain development. Lab Invest. 2003;83:479-89.

23. Tang X, Zhou L, Wagner AM, Marchetto MC, Muotri AR, Gage FH, Chen G. Astroglial cells regulate the developmental timeline of human neurons differentiated from induced pluripotent stem cells. Stem Cell Res. 2013;11:743-57.

24. Imaizumi Y, Okada Y, Akamatsu W, Koike M, Kuzumaki N, Hayakawa H, Nihira T, Kobayashi T, Ohyama M, Sato S, et al. Mitochondrial dysfunction associated with increased oxidative stress and alpha-synuclein accumulation in PARK2 iPSC-derived neurons and postmortem brain tissue. Mol Brain. 2012;5:35.

25. Falk A, Koch P, Kesavan J, Takashima Y, Ladewig J, Alexander M, Wiskow O, Tailor J, Trotter M, Pollard S, et al. Capture of neuroepithelial-like stem cells from pluripotent stem cells provides a versatile system for in vitro production of human neurons. PLoS One. 2012;7:e29597.

26. Cai J, Li W, Su H, Qin D, Yang J, Zhu F, Xu J, He W, Guo X, Labuda K, et al. Generation of human induced pluripotent stem cells from umbilical cord matrix and amniotic membrane mesenchymal cells. J Biol Chem. 2010;285:11227-34.

27. Friocourt G, Marcorelles P, Saugier-Veber P, Quille ML, Marret S, Laquerriere A. Role of cytoskeletal abnormalities in the neuropathology and pathophysiology of type I lissencephaly. Acta Neuropathol. 2011;121:149-70.

28. Gasser UE, Hatten ME. Neuron-glia interactions of rat hippocampal cells in vitro: glial-guided neuronal migration and neuronal regulation of glial differentiation. J Neurosci. 1990;10:1276-85.

29. Saillour Y, Broix L, Bruel-Jungerman E, Lebrun N, Muraca G, Rucci J, Poirier K, Belvindrah R, Francis F, Chelly J. Beta tubulin isoforms are not interchangeable for rescuing impaired radial migration due to Tubb3 knockdown. Hum Mol Genet. 2014;23:1516-26.

30. Schatz PJ, Solomon F, Botstein D: Genetically essential and nonessential alphatubulin genes specify functionally interchangeable proteins. Mol Cell Biol. 1986; 6:3722-733.

31. Tian G, Kong XP, Jaglin XH, Chelly J, Keays D, Cowan NJ. A pachygyriacausing alpha-tubulin mutation results in inefficient cycling with CCT and a deficient interaction with TBCB. Mol Biol Cell. 2008;19:1152-61.

32. Stoner R, Chow ML, Boyle MP, Sunkin SM, Mouton PR, Roy S, Wynshaw-Boris A, Colamarino SA, Lein ES, Courchesne E. Patches of disorganization in the neocortex of children with autism. N Engl J Med. 2014;370:1209-19.

33. Abuhatzira $L$, Shemer $R$, Razin $A$. MeCP2 involvement in the regulation of neuronal alpha-tubulin production. Hum Mol Genet. 2009;18:1415-23.

34. Fallet-Bianco C, Loeuillet L, Poirier K, Loget P, Chapon F, Pasquier L, Saillour Y, Beldjord C, Chelly J, Francis F. Neuropathological phenotype of a distinct form of lissencephaly associated with mutations in TUBATA. Brain. 2008;131:2304-20

35. Kato M, Dobyns WB. Lissencephaly and the molecular basis of neuronal migration. Hum Mol Genet. 2003;12(1):R89-96.

\section{Submit your next manuscript to BioMed Central and we will help you at every step:}

- We accept pre-submission inquiries

- Our selector tool helps you to find the most relevant journal

- We provide round the clock customer support

- Convenient online submission

- Thorough peer review

- Inclusion in PubMed and all major indexing services

- Maximum visibility for your research

Submit your manuscript at www.biomedcentral.com/submit
Biomed Central 